Association for Information Systems AIS Electronic Library (AISeL)

Wirtschaftsinformatik Proceedings 2003

Wirtschaftsinformatik

September 2003

\title{
Automatisierte Erkennung von marktplatzspezifischem Verhandlungsverhalten
}

Stefan Sackmann

Albert-Ludwigs-Universität Freiburg i. Br., sackmann@iig.uni-freiburg.de

Follow this and additional works at: http://aisel.aisnet.org/wi2003

\section{Recommended Citation}

Sackmann, Stefan, "Automatisierte Erkennung von marktplatzspezifischem Verhandlungsverhalten" (2003). Wirtschaftsinformatik Proceedings 2003. 12.

http://aisel.aisnet.org/wi2003/12

This material is brought to you by the Wirtschaftsinformatik at AIS Electronic Library (AISeL). It has been accepted for inclusion in Wirtschaftsinformatik Proceedings 2003 by an authorized administrator of AIS Electronic Library (AISeL). For more information, please contact elibrary@aisnet.org. 
In: Uhr, Wolfgang, Esswein, Werner \& Schoop, Eric (Hg.) 2003. Wirtschaftsinformatik 2003: Medien - Märkte - Mobilität, 2 Bde. Heidelberg: Physica-Verlag

ISBN: 3-7908-0111-9 (Band 1)

ISBN: 3-7908-0116-X (Band 2)

(C) Physica-Verlag Heidelberg 2003 


\title{
Automatisierte Erkennung von marktplatz- spezifischem Verhandlungsverhalten
}

\author{
Stefan Sackmann \\ Albert-Ludwigs-Universität Freiburg i. Br.
}

\begin{abstract}
Zusammenfassung: Der zunehmende mobile Einsatz von Informationstechnologie und die Allgegenwärtigkeit von Rechnern schaffen Informationssysteme, die durch dynamische, spontane Vernetzung und dezentrale Dienste geprägt sind. SoftwareAgenten stellen eine Technologie zur Realisierung von eingebetteten Diensten dar, die von einer Mensch-Maschine-Interaktion weitestgehend unabhängig sind. Dies erfordert ein durchgängiges Automatisierungskonzept der Transaktionsdurchführung, das auch die automatisierte Erkennung des Transaktionsumfeldes erfordert. Im vorliegenden Beitrag wird für bilaterale Preisverhandlungen ein Verfahren zur Messung des marktplatzspezifischen Verhandlungsspielraums entwickelt, in Agenten implementiert und in deren Verhandlungsverhalten integriert. Die Evaluation und Bewertung des Messverfahrens wird anhand von Simulationsreihen auf einem prototypischen elektronischen Marktplatz vorgenommen und gezeigt, dass für Agenten eine Anpassung des eigenen Verhandlungsverhaltens an das marktplatzspezifische Verhandlungsverhalten erreicht werden kann.
\end{abstract}

Schlüsselworte: Software-Agenten, bilaterale Preisverhandlung, MultiagentenSystem, Mobilität, Ubiquitous Computing

\section{Bilaterale Preisverhandlungen auf elektronischen Märkten}

Zwei technische Entwicklungstendenzen besitzen das Potential, die Realisierung und den Einsatz von Informationssystemen nachhaltig zu verändern: Zum einen ermöglicht der mobile Einsatz von Informationstechnologie den menschlichen Nutzern eine persönliche Mobilität bei globaler Erreichbarkeit, zum anderen führt die zunehmende Miniaturisierung verbunden mit sinkenden Preisen zu einer unaufhaltsamen Integration von Rechnern in Alltagsgegenstände - eine Vision, die inzwischen untrennbar mit den Begriffen „Ubiquitous Computing“, resp. „Pervasive Computing“ verbunden ist [MaLa01].

Die absehbaren Primäreffekte dieses technischen Fortschritts können in zwei Phasen unterteilt werden [Mül+02]: Zunächst werden die entstehenden Informationssysteme vor allem durch die Realisierung von Datendiensten und einen vom 
Ort unabhängigen Zugang zum Internet gekennzeichnet sein. Zunehmend werden sich die mobilen Komponenten der Informationssysteme jedoch in ihrer näheren Umgebung mit anderen mobilen oder stationären Komponenten spontan vernetzen und damit die angebotenen und nachgefragten Dienste innerhalb der dabei entstehenden ad-hoc-Netzwerke ständig neu konfigurieren.

Diese Entwicklung wird für menschliche Nutzer eine Veränderung in der Wahrnehmung und im Umgang mit Informationstechnologie mit sich bringen, so wird bspw. die Software der einzelnen Rechner durch eine weitgehende Autonomie im Sinne von ,calm technology“ [WeBr96] verfügen. Eine hierfür viel versprechende Technologie sind Software-Agenten, die im Auftrag ihrer Besitzer autonome Handlungen vornehmen können, wie z.B. den Einkauf von Kommunikationsbandbreite oder den Fahrkartenkauf beim Einsteigen in einen Zug [Ada+03]. Der mobile Zugang zum Internet wird dann zum einen durch ortsabhängige Dienste, zum anderen durch die Verfügbarkeit so genannter eingebetteter Dienste erweitert, die im Regelfall von einer Mensch-Maschine-Interaktion vollständig unabhängig sind und über einen autonomen Entscheidungsraum verfügen.

Die Realisierung eingebetteter Dienste durch Software-Agenten in einer mobilen, dynamischen Umwelt stellen den gedanklichen Rahmen des vorliegenden Beitrags dar. Basierend auf bisherigen Forschungsergebnissen im Bereich von SoftwareAgenten und Multiagenten-Systemen wird in Abschnitt 1.1 argumentiert, dass die entstehenden Informationssysteme auf der Gesamtebene über marktliche Mechanismen selbstorganisierend koordiniert werden können und dass hierbei bilaterale Transaktionsabschlüsse eine besondere Bedeutung erlangen. In Abschnitt 1.2 richtet sich der Fokus auf bilaterale Preisverhandlung, auf die Verhandlungsphasen und die jeweiligen Anforderungen an eine automatisierte Umsetzung von Verhandlungsstrategien durch Agenten. Es wird gezeigt, dass die Wahl konkreten Verhandlungsverhaltens i.d.R. nicht absolut, sondern nur in Relation zum marktplatzspezifischen Kontext vorgenommen werden kann.

In Abschnitt 2 wird daher eine Messmethode entwickelt und vorgeschlagen, die ein formales Modell zur Einschätzung des marktplatzspezifischen Verhandlungsverhaltens verfügbar macht. Die damit gewonnenen marktplatzspezifischen Informationen können als Prognosegrundlage für zukünftige Transaktionen genutzt werden und bieten zudem eine Möglichkeit, das Verhandlungsverhalten eines Transaktionspartners auf der Basis empirischer Beobachtungen zu schätzen, sowie das eigene Verhandlungsverhalten automatisch anzupassen und beabsichtigte Verhandlungsstrategien umzusetzen.

In Abschnitt 3 wird die Messmethode als generisches Modul in Agenten implementiert und in deren jeweilige adaptiv-heuristische Verhandlungsstrategie integriert. Zur Evaluierung der Funktionalität und der Auswirkungen des Messverfahrens auf die Adaptivität sowie den wirtschaftlichen Erfolg der Agenten werden auf einem prototypischen elektronischen Marktplatz Simulationen durchgeführt und anhand unterschiedlicher Simulationsszenarien gezeigt, dass durch die Inte- 
gration des Messverfahrens in die Verhandlungsstrategie eine Anpassungsfähigkeit an das auf einem konkreten Marktplatz vorherrschende „typische“ Verhandlungsverhalten erreicht werden kann.

\subsection{Marktliche Mechanismen zur Koordination von Software- Agenten - Die Bedeutung bilateraler Verhandlungen}

Die Gesamtkoordination der dezentral interagierenden, autonomen Komponenten in solchen Systemen ist eine weitestgehend offene Frage. Einen viel versprechenden Ansatz hierfür stellt die Selbstorganisation der Komponenten dar, bspw. durch die Übertragung marktlicher Mechanismen in technische Domänen [Eym+03]. Dieser erste Ansatz wird zudem durch die Erwartung unterstützt, dass die Mehrheit der in einem solch offenen Netzwerk angebotenen Dienste langfristig nur gegen Entgelt zur Verfügung stehen wird und von den Nutzern entsprechend dem erzeugten Mehrwert vergolten werden muss [MaVa95].

In der Volkswirtschaftslehre wird zur Analyse marktlicher Preismechanismen meist eine „top-down"-gerichtete analytische Vorgehensweise gewählt. Konkurrierende Transaktionsabschlüsse erhalten unter den getroffenen Annahmen ${ }^{1}$ i.d.R. eine herausragende Stellung und werden bilateralen Transaktionsabschlüssen aufgrund ihrer Optimalität als Preismechanismen vorgezogen [NeVu01]. Die Implementation von Preismechanismen, die ein Erreichen analytisch abgeleiteter Koordinationsergebnisse sicherstellen könnten, ist für das hier relevante offene, dynamische und dezentrale Szenario sowie bei begrenzter Rationalität und potentiell opportunistischem Verhalten der Akteure (vgl. [PiDi90]) - unabhängig ob Mensch oder Software-Agent - nicht direkt möglich.

Marktliche Koordination ergibt sich vielmehr indirekt und emergent durch die Aktionen der Marktteilnehmer, die nicht per se ein gemeinsames Ziel verfolgen und einer zentralen Instanz nicht vorbehaltlos vertrauen. ${ }^{2}$ Eine solche selbstorganisierende marktliche Koordination kann für elektronische Märkte jedoch nicht ,geplant“ implementiert werden. Hier können lediglich Rahmenbedingungen definiert werden, innerhalb derer die Agenten ihre vorgegebenen Ziele zu erreichen versuchen und so auf der Gesamtebene im günstigen Fall zu einer - wie auch immer gearteten - ,spontanen Ordnung" finden [Eym+03].

Für die Implementation marktlicher Preismechanismen zur Koordination von Informationssystemen bedeutet dies, dass die herausragende Stellung der analytisch optimalen, konkurrierenden Transaktionsabschlüsse nicht mehr per se gegeben

Bspw. wird oftmals die kostenlose Verfügbarkeit eines Intermediärs vorausgesetzt.

Ein hierfür adäquates Marktverständnis, das den Blick auf Anpassungsprozesse innerhalb eines Marktes richtet, findet sich in der so genannten „Österreichischen Schule“ und deren Weiterentwicklungen, bspw. in dem von Hayek entwickelten KatallaxieKonzept und der sich daraus ergebenden ,,spontanen Ordnung“ [Haye96]. 
ist. ${ }^{3}$ Im Kontext einer Umwelt mit mobilen, sich spontan vernetzenden Rechnern besitzen bilaterale Transaktionsabschlüsse Vorteile, die ihre Verwendung trotz potentiell sub-optimaler Ergebnisse, trotz relativ hoher Transaktionskosten und trotz der unter Umständen zeitintensiven Planung und Verhandlungsführung - die durch Automatisierung erheblich reduziert werden kann - aus ökonomischer Sicht sinnvoll erscheinen lassen [Sack03]: Bilaterale Transaktionsabschlüsse...

- können bereits von einem Anbieter und einem Nachfrager zum Abgleich ihrer Interessen eingesetzt werden,

- benötigen weder einen zentralisierten Marktplatz noch einen Zugang zu einer globalen Informationsinfrastruktur, da sie dezentral, direkt und in Echtzeit durchgeführt werden können,

- sind zudem für Transaktionsabschlüsse zwischen prinzipiell gleichberechtigten Marktakteuren geeignet und bevorzugen keine Marktseite per se,

- ermöglichen einen Schutz der Privatheit und gegen opportunistisch handelnde Marktakteure, da sie keine Offenlegung weder der Präferenzstruktur noch der maximalen Zahlungsbereitschaft voraus setzen,

- basieren auf zeitraumbezogenen, verbindlichen und an den jeweiligen Transaktionspartner gebundenen Angeboten und ermöglichen daher eine gewisse Planung und ad-hoc Durchführung von Transaktionen,

- sind flexibel und nicht allein auf Preisverhandlungen beschränkt.

Zusammenfassend lässt sich feststellen, dass bilaterale Transaktionsabschlüsse eine Vielzahl ,praktischer Vorteile“ in sich vereinen und es ist daher anzunehmen, dass diese im betrachteten Szenario eine bedeutende Rolle einnehmen werden [Wino02]. Ein damit verbundener Preismechanismus ist die bilaterale Verhandlung - im einfachsten Falle die bilaterale Preisverhandlung - die daher zum zentralen Betrachtungsgegenstand des vorliegenden Beitrages wird.

\subsection{Automatisierte Preisverhandlungen mit Software-Agenten}

Preisverhandlungen können allgemein in zwei Phasen unterteilt werden. Die erste Phase ist die Verhandlungseröffnung als Teil der Anbahnungsphase einer Transaktion, die zweite Phase ist die Verhandlungsführung als Teil der Kontraktoptimierung, resp. der Vereinbarungsphase (vgl. [Schm93]). Eine durchgängige Automatisierung bilateraler Preisverhandlungen ist für Agenten nur dann möglich, wenn sie ein formales, mikroökonomisches Modell für ihre Entscheidungsfindung und Aktionswahl in beiden dieser Phasen einsetzen können. Während für die Vereinbarungsphase und die Verhandlungsführung eine Vielzahl spieltheoretischer

3 Die Implementation solcher Preismechanismen wird bspw. im Market Oriented Programming (MOP) angestrebt (vgl. z.B. [Well96]). 
und adaptiv-heuristischer Einzelkonzepte existiert, fehlen für die vorgelagerte Verhandlungseröffnung sowohl in der Theorie, als auch in der Praxis, entsprechende Modelle. ${ }^{4}$

Die Verhandlungseröffnung erfordert von den Verhandlungspartnern die Bestimmung ihrer Initialangebote, durch die die Größe des Verhandlungsspielraums und die möglichen Strategien in der anschließenden Verhandlungsphase determiniert werden. Die wesentliche Grundlage hierfür ist das Verhandlungsziel des Agenten sowie Informationen über den konkreten Verhandlungspartner ${ }^{5}$ und das aktuelle Transaktionsumfeld [Lew+97]), wie bspw. das bisherige Marktgeschehen und realisierte Transaktionspreise. So sollte die Höhe des jeweiligen Eröffnungsangebots dem typischen Verhandlungsspielraum des relevanten Transaktionsumfeldes angepasst sein und unterschiedlich ausfallen, je nachdem ob um eine kleine Marge verhandelt wird oder ob sich die Verhandlungspartner auf einer Art „orientalischem Basar" treffen.

Da eine Einschätzung des marktplatzspezifischen Verhandlungsverhaltens bei bisherigen Implementationen von Agenten nicht automatisiert vorgenommen wird, stellt sie eine Lücke für die durchgängige Automatisierung der Transaktionsdurchführung dar, deren Bedeutung mit der fortschreitenden Mobilität von Informationssystemkomponenten zunehmen wird. Das hier vorgestellte Messverfahren und dessen Integration in die Verhandlungsstrategie von Agenten stellt einen ersten Schritt dar, diese zu schließen. Für die Entwicklung einer Messmethode, wie sie in Abschnitt 2 vorgeschlagen wird, gilt es zunächst, die relevanten Parameter zu identifizieren, die bezüglich des Verhandlungsergebnisses Relevanz besitzen, eine Basis für die Strategiewahl bieten sowie quantifizierbar sind und damit zur Entscheidungsfindung von Software-Agenten genutzt werden können.

Als erfolgsversprechende Parameter bieten sich statische Referenzpunkte von Preisverhandlungen an, wie der Reservations-, Ziel- und Transaktionspreis sowie das gegnerische Initialangebot. Die Berücksichtigung dieser Größen bei der Erstellung eines Initialangebots steht in einem signifikanten Zusammenhang zum Verhandlungsergebnis und -erfolg [PoBu01]. Poucke und Buelens zeigen in ihrer experimentellen Studie, dass bereits $60 \%$ der Standardabweichung des Verhandlungsergebnisses durch statische Referenzpunkte, die bereits vor Beginn der eigentlichen Verhandlung feststehen, erklärt werden können. Für die Automatisie-

4 Die Einschätzung des marktplatzspezifischen Verhandlungsverhaltens wird in der Theorie i.d.R. als gegeben angenommen (z.B. als Pay-off-Matrix in der Spieltheorie). In der Praxis muss sie von menschlichen Akteuren vorgenommen werden, wie z.B. in den Projekten Market Maker oder Kasbah [Mae+99].

5 Für anonyme Verhandlungsverfahren, wie sie typischerweise in Märkten und auch im hier relevanten Szenario vorzufinden sind, ist aufgrund der unmöglichen Zuordenbarkeit vergangener Aktionen $\mathrm{zu}$ den einzelnen Akteuren in der Informationsphase lediglich ein Rückgriff auf indirekte Methoden der Informationsgewinnung möglich, bspw. auf spieltheoretische Konzepte oder statistische Verfahren [KeLo01]. 
rung bilateraler Preisverhandlungen ist dieses Ergebnis und die daraus gezogenen Schlüsse von besonderer Relevanz, da statische Referenzpunkte eine Komplexitätsreduktion bei der Implementierung ermöglichen und verhältnismäßig einfach in ein mikroökonomisches Entscheidungsmodell integriert werden können.

\section{Der marktplatzspezifische Verhandlungsspielraum}

Das vorgeschlagene Messverfahren zur Messung des marktplatzspezifischen Verhandlungsspielraums ${ }^{6}$ basiert auf historischen, objektiv messbaren Daten des beobachteten Marktplatzes, wie Initial- und realisierten Transaktionspreisen. Die Aufarbeitung dieser Daten mittels statistischer Methoden bietet den Akteuren ein Prognosewerkzeug für zukünftig zu erwartendes Verhandlungsverhalten, das nicht auf vollständige Informationen angewiesen ist und dezentral, d.h. alleine auf den Erfahrungen eines einzelnen Akteurs basierend, eingesetzt werden kann.

\subsection{Messung des Verhandlungsspielraums einer Transaktion}

Der absolute Verhandlungsspielraum $A_{i}^{X}$ einer erfolgreichen Verhandlung kann ex post für beide Verhandlungspartner ( $K=$ Käufer, $V=$ Verkäufer) als Differenz zwischen dem jeweiligen Initialangebot $p_{i}^{t, X}$ und dem erreichten Transaktionspreis $p_{i}^{T}$ entsprechend Definition (1) berechnet werden:

$$
A_{i}^{X} \equiv\left|p_{i}^{I, X}-p_{i}^{T}\right| \quad \text { mit } X \in\{K, V\}
$$

Während für die Verhandlungsphase einer konkreten Verhandlung der absolute Verhandlungsspielraum von zentraler Bedeutung ist, basiert die angestrebte Quantifizierung marktplatzspezifischer Verhandlungsspielräume auf relativen Größen. Die relativen Verhandlungsspielräume $S_{i}^{X}$, bezogen auf den jeweiligen Transaktionspreis, werden entsprechend der Definition (2) berechnet und geben an, um welchen Anteil das Initialangebot über-, bzw. unterhalb des durch die Verhandlung erreichten Transaktionspreises $\left(p_{i}^{T} \hat{=} 100 \%\right)$ gelegen hat.

$$
S_{i}^{X} \equiv \frac{\left|p_{i}^{I, X}-p_{i}^{T}\right|}{p_{i}^{T}} \quad \text { mit } X \in\{K, V\}
$$

Ist die Bedingung $p_{i}^{I, V} \geq p_{i}^{T} \geq p_{i}^{I, K}>0$ erfüllt, dann gilt für den relativen Verhandlungsspielraum des Verkäufers $S_{i}^{V} \geq 0$ und für den des Käufers $1 \geq S_{i}^{K} \geq 0$.

$6 \quad$ Vertiefend siehe [Sack03]. 


\subsection{Messung des marktplatzspezifischen Verhandlungsspiel- raums}

Die im vorherigen Abschnitt vorgestellte Messgröße Verhandlungsspielraum bezieht sich auf jeweils eine einzelne, konkrete Verhandlung. Die Gesamtheit der auf einem Marktplatz durchgeführten Verhandlungen charakterisiert das für einen Marktplatz typische Verhandlungsverhalten. Die für einzelne Verhandlungen $e x$ post ermittelten Werte des Verhandlungsspielraums bilden eine Menge von Merkmalswerten, die mittels statistischer Verfahren beschrieben werden können und Aussagen über den marktplatzspezifischen Verhandlungsspielraum ermöglichen.

Der marktplatzspezifische Verhandlungsspielraum kann bspw. als arithmetisches Mittel aller Initialangebote der jeweiligen Marktseite berechnet werden. Aus ökonomischer Sicht ist bei rational handelnden Marktakteuren jedoch zu erwarten, dass für den Fall von Transaktionspreisen mit geringer Streuung - wie sie in einem Markt typischerweise vorliegen-zwischen dem marktplatzspezifischen Verhandlungsspielraum und dem Transaktionspreis ein funktionaler Zusammenhang besteht. Bei Transaktionspreisen oberhalb des Marktpreises ist demnach zu erwarten, dass der Käufer einen Großteil des Verhandlungsspielraums an den Verkäufer im Verlaufe der Verhandlung als Konzession abgegeben hat, d.h. der individuelle Verhandlungsspielraum aus der Sicht des Käufers größer ist, als aus der Sicht des Verkäufers $\left(S_{i}^{K}>S_{i}^{V}\right)$. Bei Transaktionspreisen unterhalb ist entsprechend das Gegenteil zu erwarten. ${ }^{7}$

Ein Messverfahren für den marktplatzspezifischen Verhandlungsspielraum sollte daher einen solchen Zusammenhang in einer adäquaten Form berücksichtigen, da diesbezügliche Kenntnisse in der Einschätzung konkreter Verhandlungssituationen wertvolle Informationen darstellen. Eine erste Näherung bietet hierfür die formale Beschreibung anhand einer linearen Funktion $y=b+m \cdot x$. So wird für den Verkäufer der Verhandlungsspielraum $\bar{S}^{V}$ in Abhängigkeit vom Transaktionspreis entsprechend der Definition (3) und analog für den Käufer $\bar{S}^{K}$ entsprechend der Definition (4) formalisiert.

$$
\begin{aligned}
& \bar{S}^{V}\left(p^{T}\right) \equiv b_{1}+m_{1} \cdot p^{T} \\
& \bar{S}^{K}\left(p^{T}\right) \equiv b_{2}+m_{2} \cdot p^{T}
\end{aligned}
$$

Die Parameter der Regressionsfunktionen werden nach der Methode der kleinsten Quadrate bestimmt. Die Wertebereiche für den individuellen Verhandlungsspiel-

\footnotetext{
In den durchgeführten experimentellen Simulationen (siehe Abschnitt 3) konnte die Existenz eines solchen Zusammenhangs festgestellt werden.
} 
raum finden auch hier ihre formale Berücksichtigung, demnach gelten für die Werte der Regressionsgeraden folgende Grenzen:

$$
\begin{aligned}
& S^{V}\left(p^{T}\right)=\max \left\{0, \bar{S}^{V}\left(p^{T}\right)\right\} \\
& S^{K}\left(p^{T}\right)=\max \left\{0, \min \left\{\bar{S}^{K}\left(p^{T}\right), 1\right\}\right\}
\end{aligned}
$$

Die Merkmalsmenge der für beide Verhandlungspartner ex post ermittelten Werte des relativen Verhandlungsspielraums werden für die grafische Darstellung in ein Koordinatensystem mit den Transaktionspreisen auf der Abszisse und den relativen Verhandlungsspielräumen auf der Ordinate übertragen. ${ }^{8}$ In Abbildung 1 ist für jede erfolgreich abgeschlossene Transaktion der dazugehörige relative Verhandlungsspielraum des Verkäufers $S_{i}^{V}$ als Merkmalswert abgetragen, in Abbildung 2 analog der jeweilige Verhandlungsspielraum des Käufers $S_{i}^{K}$. In beiden Abbildungen sind zusätzlich zu den einzelnen Merkmalswerten der marktplatzspezifische Verhandlungsspielraum der Verkäufer $S^{V}\left(p^{T}\right)$ und der Käufer $S^{K}\left(p^{T}\right)$ in Abhängigkeit vom Transaktionspreis als Regressionsgeraden eingezeichnet.

Die Messung des marktplatzspezifischen Verhandlungsverhaltens stellt eine eigenständige modulare Einheit dar (vgl. [Krau01]), die prinzipiell in jedem mikroökonomischen Verhandlungsmodell und in jeder Implementation einer Verhandlungsstrategie berücksichtigt werden kann. Aufgrund der einfachen Berechnungsverfahren ist die Messmethode für eine Automatisierung von bilateralen Preisverhandlungen und für die Implementation in Software-Agenten geeignet und könnte auch als Basisdienst auf elektronischen Marktplätzen angeboten werden.

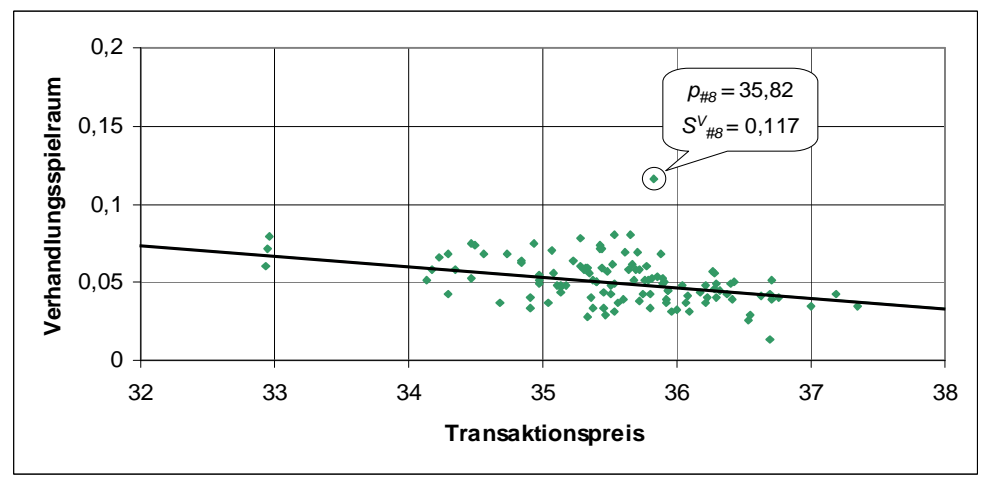

Abbildung 1: Marktplatzspezifischer Verhandlungsspielraum (Verkäufer)

8 Die den folgenden Abbildungen zugrunde liegenden Merkmalswerte sind dem Musterbeispiel in [Sack03] entnommen. 


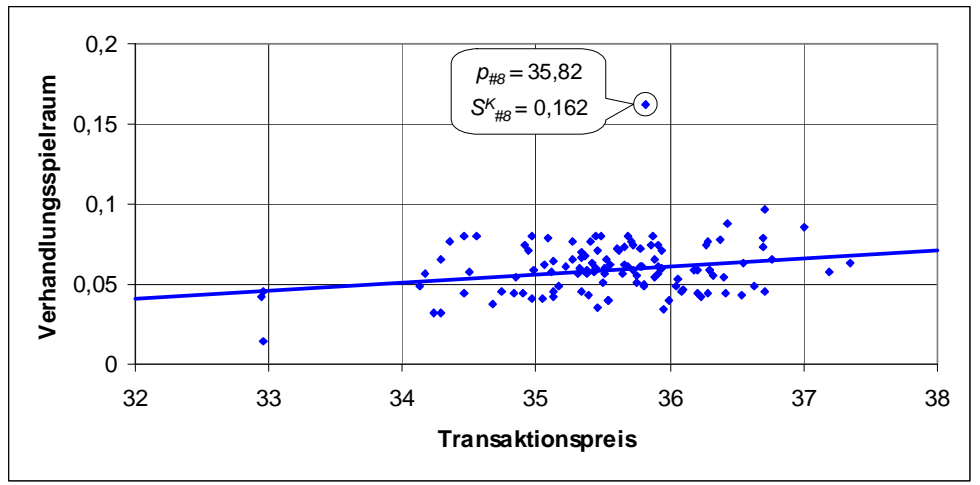

Abbildung 2: Marktplatzspezifischer Verhandlungsspielraum (Käufer)

\subsection{Berücksichtigung des Messverfahrens im mikroökono- mischen Modell von Software-Agenten}

Das vorgestellte Messverfahren für den marktplatzspezifischen Verhandlungsspielraum wurde mit dem Ziel entwickelt, wesentliche charakteristische Eigenschaften eines Marktplatzes formal beschreiben und damit ein Prognoseinstrument für verhandlungsrelevante Entscheidungssituationen zur Verfügung stellen zu können. Ausgehend von der Annahme, dass auf einem Marktplatz das zukünftig zu beobachtende Verhandlungsverhalten nicht vollkommen unabhängig vom bisher beobachteten Verhandlungsverhalten ist, können die ex post ermittelten Merkmalswerte ex ante dem Eintritt in eine konkrete Verhandlung zur Prognose des zu erwartenden Verhandlungsverhaltens genutzt werden und somit als eine Entscheidungsbasis für die Determinierung von Initialangeboten und die konkrete Verhandlungsführung eingesetzt werden.

In diesem Abschnitt wird ein mikroökonomisches Entscheidungsmodell für Preisverhandlungen vorgestellt, das zunächst von einem Akteur ausgeht, der sich konform zum relevanten Marktplatz verhält. Ausgehend von dieser ,prototypischen" Strategie können beliebige Variationen abgeleitet werden. Ein Verkäufer kann ein hohes Initialangebot bspw. durch eine Vervielfachung seiner Differenz zwischen dem Verhandlungsziel und dem errechneten marktplatztypischen Initialangebot realisieren, allerdings benötigt er auch hierfür eine Vorstellung bezüglich des marktplatzspezifischen Verhandlungsverhaltens.

\subsubsection{Eröffnung einer Verhandlung - Das Initialangebot}

Die Eröffnung einer bilateralen Preisverhandlung erfolgt durch die Abgabe eines Initialangebots. Grundlage für dessen Bestimmung bilden die ermittelten Regres- 
sionsfunktionen ${ }^{9}$ zur Prognostizierung des marktplatzspezifischen Verhandlungsverhaltens. Durch Umformung lassen sich die Initialpreise wie folgt berechnen:

$$
\begin{aligned}
& p^{I, V} \equiv s^{V}(p)=p^{T}\left(1+S^{V}(p)\right) \\
& p^{I, K} \equiv s^{K}(p)=p^{T}\left(1-S^{K}(p)\right)
\end{aligned}
$$

Mit Hilfe dieser Definitionen kann dasjenige Initialangebot, das dem marktplatzspezifischen Verhandlungsverhalten entspricht, berechnet werden, indem der Zielpreis $p^{Z, V}$, resp. $p^{Z, K}$, den der Akteur als statischen Referenzpunkt vor Beginn der Verhandlung bereits festgelegt hat, als unabhängige Variable in die Gleichung für den marktplatzspezifischen Verhandlungsspielraum eingesetzt wird.

\subsubsection{Einstieg in eine Verhandlung - Gegenangebot vs. Abbruch}

Die Entscheidung eines Akteurs, ob er bei einem vorliegenden Initialangebot in eine Verhandlung einsteigen oder nach einem anderen Verhandlungspartner suchen soll, hängt von der Interpretation des erhaltenen Initialangebotes ab. Diese wiederum ist für einen Akteur von dessen Präferenzen, Reservations- und Zielpreis sowie von den Vorstellungen des marktplatzspezifischen Verhandlungsverhaltens abhängig. In diesem Abschnitt werden unterschiedliche Möglichkeiten aufgezeigt, wie das marktplatzspezifische Verhandlungsverhalten zur Interpretation von Initialangeboten und zur adäquaten Reaktion in Abhängigkeit von der verfolgten Verhandlungsstrategie eingesetzt werden kann.

Hat der Akteur bereits ein Initialangebot seines Verhandlungspartners erhalten, muss er sich entscheiden, ob er dieses sofort annehmen möchte, ob er die Verhandlung abbricht oder ob er ein Gegeninitialangebot erstellt und damit die eigentliche Verhandlung eröffnet. Der einfachste Fall für den Akteur ergibt sich, wenn das Initialangebot bereits besser als der angestrebte Zielpreis ist, d.h. wenn die Bedingung $p^{I, K} \geq p^{Z, V}$, resp. $p^{I, V} \leq p^{Z, K}$ erfüllt ist. Für die beiden anderen Fälle ist eine Auswertung und Interpretation des gegnerischen Initialangebots notwendig. Für die Bestimmung der Erwartungswerte der gegnerischen Referenzpunkte wird wieder auf die Regressionsgeraden zurückgegriffen und zunächst der Schritt „Initialangebot erstellen“ umgekehrt, d.h. formal die quadratischen Gleichungen (7), bzw. (8) nach dem zu erwartenden Preis aufgelöst. Der Verkäufer berechnet den erwarteten Transaktionspreis $p^{T^{*}}$, resp. den geschätzten Zielpreis des Käufers $p^{Z, K^{*}}$, anhand der Definition (9) und in Abhängigkeit vom erhaltenen Initialangebot des Käufers $p^{I, K}:{ }^{10}$

9 Die Problematik der Datenverfügbarkeit, die für die Anwendung des Messverfahrens benötigt werden, ist in [Sack03] vertiefend diskutiert.

10 Der Käufer kann den erwarteten Transaktionspreis $p^{T^{*}}$, resp. den geschätzten Zielpreis des Verkäufers $p^{Z, V^{*}}$ berechnen. Aus ökonomischer Sicht sind nur jene Lösungen 


$$
p_{1,2}^{T^{*}} \equiv p_{1,2}^{Z, K^{*}}=-\frac{1-b_{2}}{2 \cdot\left(-m_{2}\right)} \pm \sqrt{\left(\frac{1-b_{2}}{2 \cdot\left(-m_{2}\right)}\right)^{2}-\frac{p^{I, K}}{m_{2}}}
$$

In Abhängig von der Höhe des eigenen Reservations- und Zielpreises können durch den Käufer drei ökonomisch relevante Fälle unterschieden werden:

1. Fall: $p^{I, V} \leq p^{Z, K}$ : Der Initialpreis des Verkäufers ist geringer als der Zielpreis des Käufers. Der Käufer sollte sich für die direkte Annahme entscheiden.

2. Fall: $p^{Z, V^{*}} \leq p^{Z, K}$ : Der erwartete Zielpreis des Verkäufers ist geringer als der Zielpreis des Käufers. Für diesen Fall ist eine leichte Verhandlung zu erwarten, bei der beide Verhandlungspartner ein gutes Ergebnis erreichen können. Die Eröffnung der Verhandlung durch ein Gegeninitialangebot sollte erfolgen.

3. Fall: $p^{Z, V^{*}}>p^{Z, K}$ : Der erwartete Zielpreis des Verkäufers liegt über dem Zielpreis des Käufers. Für diesen Fall ist eine harte Verhandlung zu erwarten und ein erfolgreicher Abschluss nur dann möglich, wenn mindestens einer der beiden Verhandlungspartner von seinem ursprünglichen Zielpreis abweicht. In diesem Fall ist eine Entscheidung notwendig, ob die Verhandlung abgebrochen oder ein Gegeninitialangebot erstellt werden soll. Eine eindeutige Handlungsempfehlung kann in diesem Fall nicht ausgesprochen werden, da die adäquate Wahl der Aktion von der verfolgten Strategie des Akteurs abhängig ist und nur in Relation zu einem konkreten Transaktionsumfeld gefunden werden kann. Mit der Messung des marktplatzspezifischen Verhandlungsspielraums und den daraus ableitbaren Referenzpunkten steht hierfür eine formale Methode zur Verfügung. Während die Meßmethode unabhängig von der konkreten Strategie eines Akteurs zur Analyse des marktplatzspezifischen Verhandlungsverhaltens genutzt werden kann, ist deren Berücksichtigung bei der Entscheidung für ein konkretes Verhandlungsverhalten nicht mehr unabhängig von der Strategie des Akteurs möglich. ${ }^{11}$

\section{Implementation und Evaluation der Messmethode}

Für die Bewertung des vorgeschlagenen Messverfahrens wurde ein prototypischer elektronischer Marktplatz implementiert, das Messverfahren in eine heuristische Verhandlungsstrategie integriert und als internes Modell eines Software-Agenten implementiert. Die software-technische Basis für die zur Bewertung durchgeführten Simulationen bildet das Multi-Agenten-System „B2B-OS“, das seit 1997

gültig, die einen erwarteten Preis ergeben, der oberhalb des Initialangebots liegt [Raif82]. Es muss daher die Bedingung $p^{I, K} \leq p^{Z, K^{*}} \leq p^{R, K^{*}}$ erfüllt sein. Vertiefend siehe [Sack03]. 
am Institut für Informatik und Gesellschaft, Abteilung Telematik, der AlbertLudwigs-Universität entwickelt wird und bereits in unterschiedlichen Veröffentlichungen vorgestellt wurde [Eyma02; Pad+02].

Die Software-Agenten, die für die Evaluation implementiert wurden, besitzen eine heuristisch-adaptive Strategie zur Preisfindung, die sich an den Arbeiten von Cliff [Clif97], Preist und Tol [PrTo98] sowie an dem so genannten „VariationImitation-Decision"-Modell von Brenner [Bren99] orientiert und den Ansatzpunkt für die Integration der vorgestellten Meßmethode darstellt. ${ }^{12}$ Für die Überprüfung und Anpassung der Marktpreiseinschätzung nutzen die Agenten jede Preisinformation, die sie durch eine erfolgreiche oder abgebrochene Verhandlung erhalten können und setzen hierfür eine modifizierte ,derivative-following“-Strategie [GrKe01] ein. Die Agenten werden dahingehend programmiert, dass sie bei einer erfolgreichen Verhandlung den erzielten Transaktionspreis als Vorstellung des aktuellen Marktpreises direkt übernehmen und davon ausgehen, dass spätere Transaktionen mindestens zu den gleichen oder besseren Bedingungen realisiert werden können. Im Falle eines Verhandlungsabbruchs geht das letzte gültige Angebot des Verhandlungspartners, zu dem eine Transaktion potentiell hätte stattfinden können, gewichtet in die Marktpreisvorstellung ein. Dieses Anpassungsverhalten entspricht einem „Versuch und Irrtum“-Vorgehen und nimmt nach jeder Transaktion eine inkrementelle Veränderung der Parameterwerte vor.

In den folgenden Abschnitten wird die Durchführung und Auswertung mehrerer Simulationsreihen beschrieben, anhand derer das Messverfahren in seiner Funktionalität und Auswirkungen bezüglich der geforderten Eigenschaften innerhalb der Grenzen der einfach gehaltenen Simulationsumgebung bewertet werden kann. In Abschnitt 3.1 wird das Referenzszenario vorgestellt, in dem keiner der Agenten das Messverfahren einsetzt. In Abschnitt 3.2 wird in einem ersten Schritt die Funktionalität des Messverfahrens überprüft, in einem zweiten Schritt das Adaptionsverhalten der Agenten analysiert und in einem dritten Schritt die Auswirkungen auf die Koordination des Gesamtsystems betrachtet.

\subsection{Das Referenzszenario ohne Berücksichtigung des markt- platzspezifischen Verhandlungsverhaltens}

Im Referenzszenario werden auf jeder Marktseite 12 Software-Agenten gestartet, die sich als potentielle Verhandlungspartner gegenüber stehen und ihren Initialpreis jeweils moderat über bzw. unter ihrer aktuellen Vorstellung des Marktpreises festlegen. Die erste Simulation erfolgt mit ,normaler“ Parametersetzung der Agenten, d.h. sie erhalten alle zu Beginn der Simulation eine Vorstellung des

12 Die Strategie wurde bereits im MAS Avalanche benutzt und trägt daher dessen Namen. Eine detaillierte Beschreibung der Strategie-Parameter und Anmerkungen zur konkreten Implementation finden sich in [Eyma02]. 
aktuellen Marktpreises. Käufer glauben das Gut für 30 Einheiten kaufen zu können, Verkäufer für 40 Einheiten verkaufen zu können. Die Reservationspreise der Käufer und Verkäufer werden so gesetzt, dass für jede Verhandlung ein positiver Verhandlungsraum existiert, so gehen die Käufer nicht über 60 Einheiten, die Verkäufer lassen sich nicht unter 20 Einheiten herunter handeln.

In Abbildung 3 ist ein einzelner Simulationslauf über die Zeit exemplarisch abgebildet. Die Agenten können 250 Sekunden lang Verhandlungen durchführen und Transaktionen abschließen. Jeder Punkt entspricht einer abgeschlossenen Transaktion zum entsprechenden Preis. In dem dargestellten Simulationslauf wurden insgesamt 713 Transaktionen durchgeführt, der durchschnittliche Transaktionspreis betrug 35,21 Einheiten mit einer Standardabweichung von 0,98.

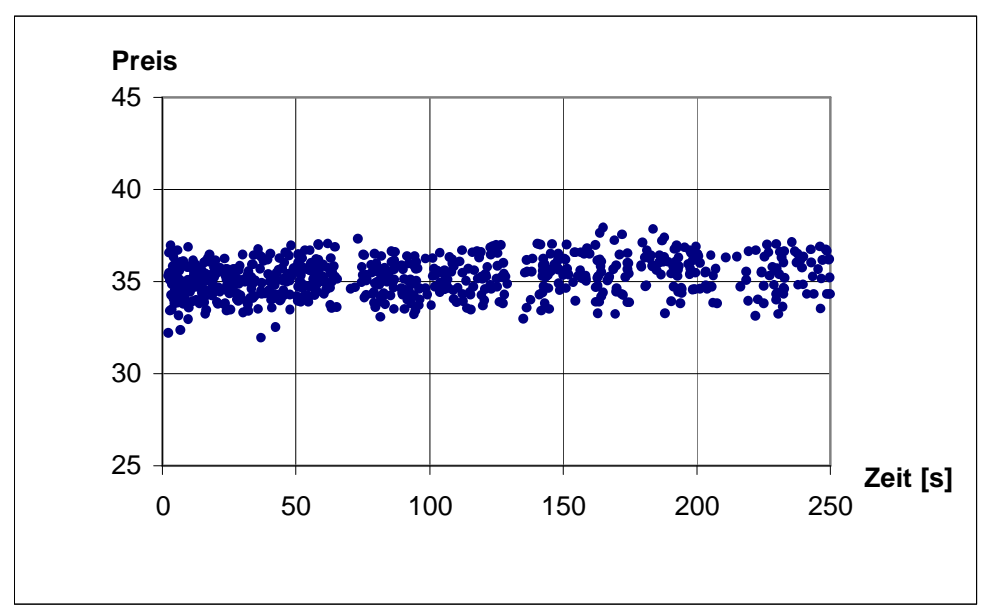

Abbildung 3: Typischer Simulationslauf im Multiagenten-System B2B-OS

Für die Überprüfung des Multi-Agenten-Systems kann festgestellt werden, dass auf der Gesamtebene wie erwartet eine Koordination alleine aus den Interaktionen und ohne zentrale Koordinationsinstanz emergent zustande kommt. ${ }^{13}$

\subsection{Integration des Messverfahrens in Software-Agenten}

In einem zweiten Schritt gilt es, die Funktionalität des Messverfahrens zu überprüfen und dessen Integration in das Verhandlungsverhalten der Agenten zu bewerten. Diese Evaluation erfolgt anhand von drei aufeinander aufbauenden Szenarien. Zunächst wird in Abschnitt 3.2.1 gezeigt, dass das Messverfahren dezentral ein-

13 Für die Überprüfung der Stabilität der Ergebnisse anhand von Simulationsreihen und unterschiedlichen Simulationsszenarien („Basar“, „Fixpreissystem“, „heterogene Strategien“, „Zufall“) sei vertiefend auf [Sack03] verwiesen. 
gesetzt werden kann, um das auf der makroökonomischen Ebene zu beobachtende Verhandlungsverhalten der Verhandlungspartner zu beschreiben. In Abschnitt 3.2.2 wird gezeigt, dass sich ein Agent durch den Einsatz des vorgeschlagenen Messverfahrens bereits nach wenigen Transaktionen an das marktplatzspezifische Verhandlungsverhalten eines unbekannten Marktplatzes anpassen kann und sich diese Fähigkeit positiv auf seinen ökonomischen Erfolg auswirkt. In Abschnitt 3.2.3 wird gezeigt, dass die Koordinationsleistung des Marktplatzes auch für den allgemeinen Einsatz des Messverfahrens von allen Agenten weiterhin erreicht werden kann und es nicht zu einem Zusammenbruch der Koordination kommt.

\subsubsection{Dezentraler Einsatz des Messverfahrens}

Die erste Simulationsreihe zur Überprüfung der Funktionalität des Messverfahrens bei dezentralem Einsatz entspricht in ihrer Konfiguration dem in Abschnitt 3.1 beschriebenen Referenzszenario. Lediglich einer der 12 Käufer-Agenten wird in seiner „Intelligenz“ erweitert und setzt das Messverfahren zur Schätzung der Referenzpunkte des Verhandlungspartners ein. Agenten, die auf das Messverfahrens zurückgreifen, verhalten sich zu den anderen Agenten so lange identisch, bis sie genügend „Verhandlungserfahrung“ und Merkmalswerte gesammelt haben. Ab dem Zeitpunkt, in dem das Messverfahren für die Einschätzung des marktplatzspezifischen Verhandlungsverhaltens eingesetzt werden kann, ändern sie ihr Verhandlungsverhalten in zwei Punkten: Erstens wird die Höhe der Initialangebote durch das in Abschnitt 2.2 beschriebene Berechnungsverfahren ermittelt. Zweitens wird die Erstellung eines Gegeninitialangebots nicht mehr unabhängig von einem erhaltenen Initialangebot bestimmt, sondern berücksichtigt dieses bei der Wahl der Reaktion und der Bestimmung der Höhe des Gegeninitialangebots.

Besteht die Datenbasis eines Käufers ${ }^{14}$ ausschließlich aus der eigenen Transaktionshistorie, dann kann eine Einschätzung des marktplatzspezifischen Verhandlungsverhaltens der Käufer insgesamt durch einen Käufer nicht direkt erfolgen und damit ist eine dezentrale Einschätzung der eigenen Marktseite ohne zusätzliche Informationen nur unzureichend möglich. ${ }^{15} \mathrm{Im}$ Gegensatz zur eigenen Marktseite kann die Einschätzung der gegenüberliegenden Marktseite jedoch direkt vorgenommen werden. In Abbildung 4 sind „Momentaufnahmen“ der geschätzten Regressionsgeraden bezüglich des Verhandlungsverhaltens der Verkäufer durch die Käufer dargestellt. Die durchgezogene Linie repräsentiert die für alle

14 In den folgenden Abschnitten wird aus Darstellungsgründen ausschließlich die Seite des Käufers betrachtet und ausgewertet, die gezeigten Ergebnisse können analog auf die Verkäufer übertragen werden.

15 Das Verhandlungsverhalten der anderen Käufer kann ohne zusätzlichen Informationsaustausch lediglich indirekt über die Reaktionen der Verkäufer auf das eigene Verhandlungsverhalten beobachtet und eingeschätzt werden. Für eine Diskussion der sich daraus ergebenden „Lock in“-Effekte und mögliche Gegenmaßnahmen sei wiederum auf [Sack03] verwiesen. 
Transaktionen des Marktplatzes berechnete Funktion. Die gestrichelte Linie stellt die vom Käufer-Agenten dezentral berechnete, ausschließlich auf den eigenen Transaktionserfahrungen basierende Einschätzung des marktplatzspezifischen Verhandlungsverhaltens der Verkäufer dar. Die deutlich erkennbare Annäherung des dezentralen Messergebnisses an das auf dem gesamten Markplatz beobachtbare Verhandlungsverhalten konnte in den durchgeführten Simulationsläufen durchgängig beobachtet werden.
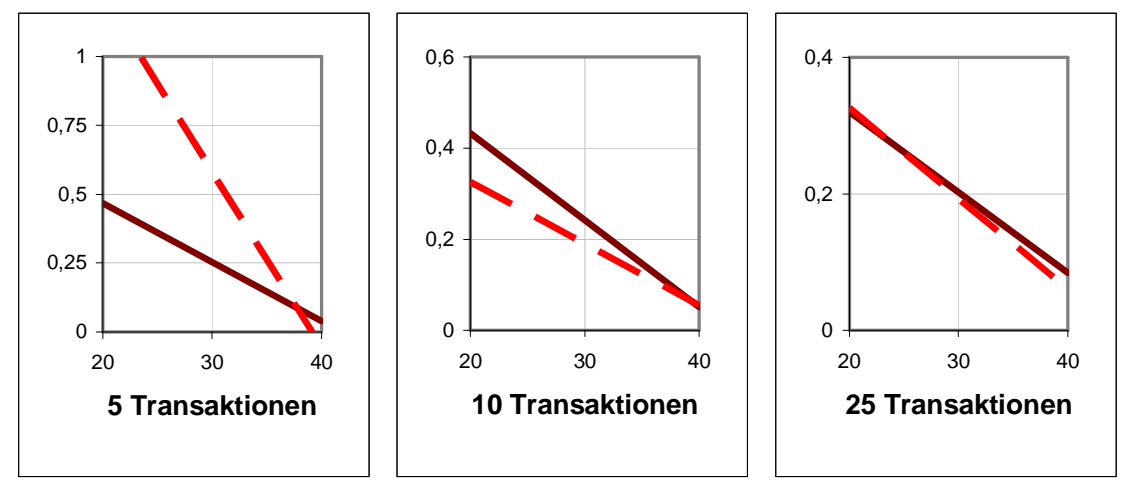

Abbildung 4: Dezentrale Messung des marktplatzspezifischen Verhandlungsraums Einschätzung der Verkäufer durch einen Käufer

Das vorgeschlagene Messverfahren bietet den Agenten somit eine Möglichkeit, von einem erhaltenen Initialangebot auf den Zielpreis des Verhandlungspartners, resp. den zu erwartenden Transaktionspreis, zu schließen. Die gute Einschätzung des zu erwartenden Transaktionspreises und deren Berücksichtigung in der Verhandlungsstrategie wirken sich positiv auf das Verhandlungsverhalten der Agenten aus. So werden signifikant weniger begonnene Verhandlungen abgebrochen und weniger Verhandlungsschritte für einen Transaktionsabschluss benötigt.

\subsubsection{Adaptionsverhalten bei integriertem Messverfahren in der Verhandlungsstrategie von Software-Agenten}

In einem zweiten Simulationsszenario zur Bewertung des Adaptionsverhaltens werden zwei Agenten mit einer abweichenden Parametersetzung zu den restlichen Agenten gestartet und haben beim Eintritt in den elektronischen Marktplatz eine „falsche“ Vorstellung ${ }^{16}$ vom marktplatzspezifischen Verhandlungsverhalten. Einer

16 Der marktplatzspezifische Verhandlungsspielraum liegt bei 15\%. Die Agenten mit den „falschen“ Vorstellungen gehen jedoch von einem Verhandlungsspielraum von $55 \%$ aus und setzen entsprechend ihre ,extremen“ Initialangebote. 
dieser beiden Agenten integriert das Messverfahren in sein Verhandlungsverhalten, der andere dient als Kontroll-Agent.

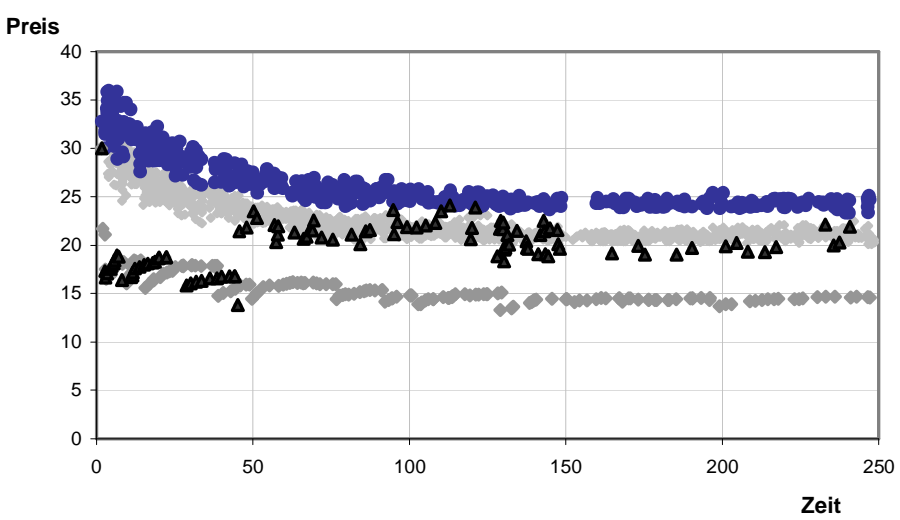

Abbildung 5: Anpassung der Initialangebote an den Marktplatz

In Abbildung 5 ist wiederum eine beispielhafte Simulation im Zeitablauf dargestellt. ${ }^{17}$ Das dunkel eingefärbte Punkteband oben repräsentiert die auf dem Marktplatz realisierten Transaktionspreise, das direkt darunter liegende hellere Punkteband die abgegebenen Initialangebote der ,normalen“ Käufer. Die Initialangebote des modifizierten Agenten sind als dunkle Dreiecke, die des Kontroll-Agenten als graue Rauten dargestellt. In mehreren Simulationsläufen zeigt sich wie erwartet, dass sich der modifizierte Agent zu Beginn der Simulationen in seiner Bestimmung der Initialangebote vom Kontroll-Agenten nicht unterscheidet.

Nach ca. 50 Sekunden hat der modifizierte Agent ausreichend Merkmalswerte gesammelt, kann das marktplatzspezifische Verhandlungsverhalten messen und zur Bestimmung der Initialangebote nutzen. Es ist deutlich zu erkennen, dass der Agent sein Verhandlungsverhalten in kurzer Zeit an das marktplatzspezifische Verhandlungsverhalten anpassen kann. Die Auswertung der Simulationsreihen zeigt zudem, dass der modifizierte Agent signifikant mehr Transaktionen abschließen kann als der Kontrollagent und weniger Verhandlungsschritte bis zu einem erfolgreichen Abschluss benötigt. Die Simulationsergebnisse lassen daher erwarten, dass durch den Einsatz des vorgeschlagenen Messverfahrens auch eine automatisierte Einschätzung des Transaktionsumfelds für Agenten oder eingebettete Dienste in offenen Systemen realisierbar ist.

17 Die Stabilisierung des Marktpreises findet je nach Simulationslauf auf unterschiedlichem Niveau statt, was auf die Heterogenität der Agenten im Bezug auf ihr Konzessionsverhalten und auch auf die „extremen“ Initialangebote zurückgeführt werden kann [Sack03]. 


\subsubsection{Gesamtkoordination bei integriertem Messverfahren in der Verhandlungsstrategie aller Software-Agenten}

Im letzten Simulationsszenario wird der Fokus auf die Makroebene gerichtet und überprüft, welche Auswirkungen der Einsatz des Messverfahrens auf einen elektronischen Marktplatz und dessen Koordinationsleistung hat, wenn ausschließlich Agenten miteinander Verhandlungen führen, die das Messverfahren nutzen. Die durchgeführten Simulationen zeigen durchgängig, dass auch in diesem Szenario eine Koordination der Agenten emergent entsteht und sich diese in ihrem Verhandlungsverhalten aufeinander einspielen und somit durch ihre Interaktion ein marktplatzspezifisches Verhandlungsverhalten erzeugen.

Ein weiteres Beispiel für die Selbstkoordination der Agenten ist in Abbildung 6 dargestellt. Auch diese Simulation wurde mit den gleichen Parameterwerten wie oben durchgeführt. Einer der Käufer-Agenten erhält aufgrund seiner ersten durchgeführten Transaktionen anfangs eine falsche Einschätzung des marktplatzspezifischen Verhandlungsverhaltens und beginnt daher ,untypische“ Initialangebote zu berechnen. Nach mehreren fehlgeschlagenen Versuchen gelingt es diesem jedoch durch zusätzliche Merkmalswerte seine Einschätzung in relativ kurzer Zeit zu korrigieren und sein Verhandlungsverhalten wieder dem der anderen Agenten anzupassen. Mit fortschreitender Simulationsdauer ergibt sich insgesamt ein stabiles marktplatzspezifisches Verhandlungsverhalten.

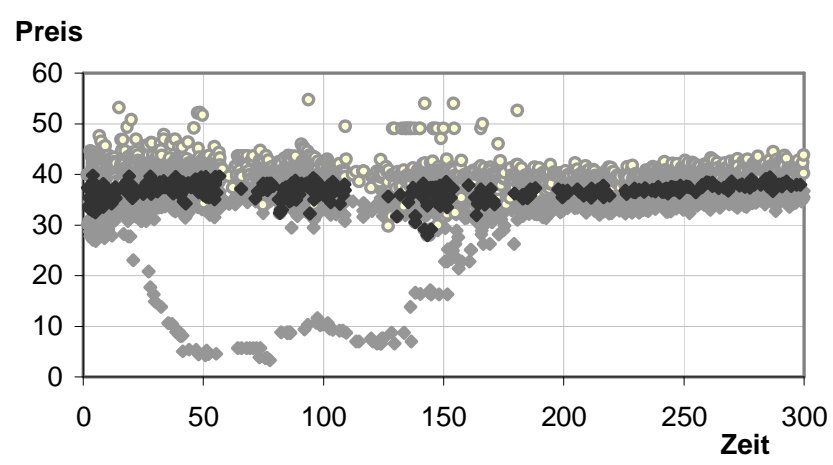

Abbildung 6: Beispiel einer „Selbstregulierung“

Die Simulationsergebnisse lassen erwarten, dass die Koordinationsleistung eines elektronischen Marktes durch die Messung und Berücksichtigung des marktplatzspezifischen Verhandlungsverhaltens durch die Agenten nicht gefährdet ist. Eine Bereitstellung der Informationen über das marktplatzspezifische Verhandlungsverhalten durch den Marktplatzbetreiber wäre demnach ein möglicher Dienst, der neu 
in das System eintretenden Agenten eine noch bessere Anpassung des eigenen Verhandlungsverhaltens ermöglichen könnte.

\subsection{Ergebnisse und Interpretation der Simulationen}

Mit den vorgestellten Simulationen konnte gezeigt werden, dass das vorgeschlagene Messverfahren dezentral zur Einschätzung des marktplatzspezifischen Verhandlungsverhaltens der gegenüberliegenden Marktseite eingesetzt werden kann. Es bietet zudem eine Möglichkeit, das Verhandlungsverhalten von Agenten auf einem elektronischen Marktplatz automatisiert zu erkennen. Bei einer entsprechenden Berücksichtigung im internen Modell kann damit das Verhandlungsverhalten von Software-Agenten automatisch an das marktplatzspezifische Verhandlungsverhalten angepasst werden.

\section{Zusammenfassung und Ausblick}

Die zunehmenden Möglichkeiten des mobilen Einsatzes von Informationstechnologie und spontaner ad-hoc-Vernetzung von Geräten eröffnet im ökonomischen Kontext die Etablierung neuartiger digitaler Dienste. Software-Agenten können ohne ständige Interaktion mit ihrem menschlichen Besitzer wirtschaftliche Transaktionen automatisiert durchführen. In den vorhergehenden Abschnitten wurde gezeigt, dass in einem offenen System die Preisverhandlungen autonom und eigennützig handelnder Agenten anhand bilateraler Verhandlungen dezentral aufeinander abgestimmt werden können.

Die automatisierte Durchführung bilateraler Verhandlungen durch SoftwareAgenten erfordert ein durchgängiges Konzept, das neben der Verhandlungsführung auch eine automatisierte Anpassung der Verhandlungsstrategie an das konkrete Transaktionsumfeld umfassen muss. Das im vorliegenden Beitrag vorgestellte Messverfahren stellt eine eigenständige, modulare Einheit dar, die es den Agenten ermöglicht, zum einen das typische Verhandlungsverhalten auf einem Marktplatz automatisch zu erkennen und einzuschätzen, zum anderen prinzipiell in jede Verhandlungsstrategie integriert werden kann.

Die Integration des Messverfahrens in ein mikroökonomisches Verhaltensmodell wird in diesem Beitrag am Beispiel einer heuristisch-adaptiven Verhandlungsstrategie beispielhaft durchgeführt und in das internes Modell von SoftwareAgenten implementiert. Aufgrund der einfachen Berechnungsverfahren, die dem Messverfahren zugrunde liegen, sind die zusätzlichen Anforderungen an die verfügbare Rechenkapazität gering und daher auch im Kontext des mobilen Einsatzes von Informationstechnologie realisierbar. 
Die experimentellen Ergebnisse stellen zwar keinen formalen Beweis für den Zusammenhang zwischen der Integration des Messverfahrens und einem wirtschaftlich erfolgreichen Handeln von Agenten dar, sie lassen jedoch eine verbesserte Einsatzmöglichkeit von Software-Agenten in offenen Systemen erwarten. Vor allem im Kontext des mobilen Einsatzes von Informationstechnologie ist das Erkennen des marktplatzspezifischen Verhandlungsverhaltens und die Anpassung der eigenen Verhandlungsstrategie für Agenten von besonderer Bedeutung, da sich durch einen Ortswechsel für die angebotenen und nachgefragten Dienste das konkrete Transaktionsumfeld ständig verändern kann. Die Integration des Messverfahrens stellt damit einen elementaren Baustein für die Realisierung mobiler, eingebetteter Dienste dar.

Die durch die technische Entwicklung entstehenden Anforderungen an eine automatisierte Durchführung von Transaktionen und die erzielten experimentellen Ergebnisse stellen einen Anlass zur weiteren Forschung dar. Es gilt in einem nächsten Schritt zu untersuchen, wie zuverlässig das in dieser Arbeit entwickelte Messverfahren außerhalb des Experimentalsystems eingesetzt werden kann. Hierfür wird das implementierte Modul in weitere Verhaltensstrategien integriert und in praktischen Anwendungen zum Einsatz kommen. Konkret geplant ist die Integration des Messverfahrens in die Projekte CatNet und EMIKA, ${ }^{18}$ die eine Evaluation des Messverfahrens außerhalb der bisher eingesetzten Simulationsumgebung ermöglichen. Sie bieten zudem die Möglichkeit zur Analyse und Weiterentwicklung des Messverfahrens selbst und zur Entwicklung eines generischen Moduls, das in einer Vielzahl unterschiedlichster Strategien integriert werden kann und in offenen Systemen eine Adaption des eigenen Verhandlungsverhaltens an konkrete, unbekannte Marktplätze ermöglicht.

Es gilt zudem zu klären, in wie weit eine Bereitstellung der Informationen über das marktplatzspezifische Verhandlungsverhalten durch eine vertrauenswürdige Instanz die Koordination der Agenten und die Koordinationsleistung des Marktplatzes auf der Makroebene positiv beeinflussen kann. Auch die Übertragbarkeit des Messverfahrens auf andere marktliche Koordinationsmechanismen, wie bspw. auf Auktionen zur Messung des „,marktplatzspezifisches Gebotsverhalten“, ist möglicher Gegenstand weiterer Forschung.

18 Das Projekt CatNet untersucht die dezentrale Koordinationsleistung von SoftwareAgenten im Kontext von ,dynamic appliation layer networks“ und wird durch die EU gefördert (Projektdetails finden sich unter http://www.telematik.uni-freiburg.de/ CatNet/index.php). Das von der DFG geförderte Projekt EMIKA strebt eine echtzeitfähige Krankenhauslogistik durch dezentrale Koordination über Software-Agenten mit Hilfe mobiler, spontan vernetzbarer Endgeräte an [Sac+02]. 


\section{Literatur}

[Ada+03] Adams, T.; Ferguson, G.; Tobolski, J. F.: An Introduction to Silent Commerce: Creating New Sources of Value from Intelligent Objects. Accenture Tech. Labs, Chicago, 2003.

[Bren99] Brenner, T.: A Behavioural Learning Approach to the Dynamics of Prices. MaxPlanck-Institut für die Erforschung von Wirtschaftssystemen, Jena 1999

[Clif97] Cliff, D.: Minimal-Intelligence Agents for Bargaining Behaviors in Market-Based Environments. Hewlett Packard Labs, Bristol, 1997

[Eyma02] Eymann, T.: Digitale Geschäftsagenten - Software-Agenten im Einsatz. Xpert.press, Berlin: Springer-Verlag, 2002.

[Eym+03] Eymann, T.; Sackmann, S.; Müller G.: Hayeks Katallaxie - Ein zukunftsweisendes Konzept für die Wirtschaftsinformatik? Schwerpunktheft der Zeitschrift Wirtschaftsinformatik (noch nicht erschienen), 2003.

[GrKe01] Greenwald, A. R.; Kephart, J. O.: Shopbots and Pricebots. Berlin: Springer Verlag, 2001.

[Haye96] Hayek, F. A.: Evolution und Spontane Ordnung, in: Hayek, F. A. (Hrsg.): Die Anmaßung von Wissen. Tübingen: Siebeck, 1996.

[KeLo01] Kersten, G. E.; Lo, G.: Negotiation Support Systems and Software Agents in EBusiness Negotiations, in: $1^{\text {st }}$ International Conference on Electronic Business, Hong Kong, 2001.

[Krau01] Kraus, S.: Strategic Negotiation in Multiagent Environments. Cambridge, Massachusetts: Bradford Book, MIT-Press, 2001.

[Lew+97] Lewicki, R. J.; Saunders, D. M.; Minton, J. W.: Essentials of Negotiation. Boston, New York, u.a.O.: Irwin, 1997.

[Mae+99] Maes, P.; Guttman , R. H.; Moukas, A.: Agents that buy and sell, in: Communications of the ACM 42 (3), 1999, S. 81-91.

[MaLa01] Mattern, F.; Langheinrich, M.: Allgegenwärtigkeit des Computers - Datenschutz in einer Welt intelligenter Alltagsdinge. Berlin: Springer, 2001.

[MaVa95] MacKie-Mason, J. K.; Varian, H.: Pricing the Internet, in: Kahin, B. and Keller, J. (Hrsg.): Public access to the Internet. Cambribge MA: MIT Press, 1995.

[Mül+02] Müller, G.; Eymann, T.; Kreutzer, M.: Telematik- und Kommunikationssysteme in der vernetzten Wirtschaft. München, Wien: Oldenbourg-Verlag, 2002.

[NeVu01] Neeman, Z.; Vulkan, N.: Market versus Negotiations: The Predominance of Centralized Markets, Working Paper, http://www.ma.huji.ac.il/ neeman/papers/ MarketsVrNego.pdf, 2001, Abruf am 2002-11-01.

[Pad+02] Padovan, B.; Sackmann, S.; Eymann, T.; Pippow, I.: A Prototype for an Agentbased Secure Electronic Marketplace including Reputation Tracking Mechanisms, in: International Journal of Electronic Commerce 6 (4), 2002, S. 93-113. 
[PiDi90] Picot, A.; Dietl, H.: Transaktionskostentheorie, in: WIST, Heft 4, April 1990, S. 178-183.

[PoBu01] Poucke, D.; Buelens, M.: Predicting the negotiated outcome - The Role of Structural Reference Points and Negotiator's Offer Zone. Vlerick Leuven Gent Management School, Belgien, 2001.

[PrTo98] Preist, C.; Tol, M. v.: Economic Agents for Automated Trading. Agent Technology Group Discussion Paper, Hewlett-Packard Labs, Bristol, UK 1998.

[Raif82] Raiffa, H.: The art and science of negotiation. Cambridge: Harvard Univ. Press, 1982.

[Sac+02] Sackmann, S.; Eymann, T.; Müller, G.: EMIKA - Real-Time Controlled Mobile Information Systems in Health Care Applications, in: Bludau, H.-B.; Koop, A. (Hrsg.): Mobile Computing in Medicine. Proceedings of 2nd Conference on Mobile Computing in Medicine. GI-Edition Lecture Notes in Informatics, Bonn: Gesellschaft für Informatik, 2002.

[Sack03] Sackmann, S.: Bilaterale Preisverhandlungen von Software-Agenten - Ein Modell und System zur Analyse des marktplatzspezifischen Verhandlungsspielraumes. Wiesbaden: Deutscher Universitäts-Verlag, Gabler (zugl. Dissertation, Albert-LudwigsUniversität Freiburg i. Br., 2002).

[Schm93] Schmid, B.: Elektronische Märkte, in: Wirtschaftsinformatik 35 (5), 1993, S. $465-480$

[WeBr96] Weiser, M.; Brown, J. S.: The Coming Age of Calm Technology. Xerox PARC, http://www.ubiq.com/hypertext/weiser/acmfuture2endnote.htm, 1996, Abruf am 200301-23.

[Well96] Wellman, M. P.: Market-Oriented Programming: Some Early Lessons, in: Clearwater, S. H. (Hrsg.): Market-Based Control: A Paradigm for Distributed Resource Allocation. Singapore: World Scientific, 1996.

[Wino02] Winoto, P.: An Extended Alternating-Offers Bargaining Protocol for Automated Negotiation in Multi-agent Systems, in: Proceedings of the 10th International Conference on Cooperative Information Systems (CoopIS'2002), Irvine, November 2002, S. 179-194. 